\title{
EDITORIAL
}

\section{Física en las Ciencias de la Salud: una necesidad apremiante}

\author{
Jorge Homero Wilches-Visbal (D) 1
}

1. Universidad del Magdalena. Santa Marta, Colombia. Correo: jhwilchev@gmail.com - https://orcid.org/0000-0003-3649-5079

La ciencia es una actividad creativa de la mente humana cuyo propósito es la búsqueda de patrones en el universo mediante la observación y no una mera colección de hechos e invención de teorías. Los patrones desvendados estimulan la concreción de modelos que son representaciones pictóricas que explican los fenómenos observados. Los modelos pueden progresar a teorías y leyes con una sólida base de predicción y reproductibilidad dentro de un rango de acción definido ${ }^{1}$.

La física es una ciencia natural y exacta relacionada con el estudio de la materia y la energía en el tejido espaciotemporal. Se considera a la física como la "ciencia fundamental" pues todas las demás ciencias naturales están supeditadas a sus leyes ${ }^{2}$.

En un sentido más amplio, la física estudia el comportamiento del universo ${ }^{3}$, desde sus estructuras microscópicas (átomos, moléculas, organismos) hasta las macroscópicas (planetas, estrellas, agujeros negros). Según la escala y naturaleza del objeto de estudio, la física se subdivide en diversas ramas: astrofísica, física nuclear, física clásica, física teórica, biofísica, entre otras.

La relevancia de la física para la humanidad fue reconocida en la Asamblea de las Naciones Unidas con motivo de la conmemoración del año mundial de la física, celebrado en 2005, expresándose, literalmente, que ${ }^{4}$ :

- "La física constituye un fundamento importante para comprender mejor la naturaleza".
- "La física y sus aplicaciones son la base de muchos de los avances tecnológicos de la actualidad".

- "La enseñanza de la física proporciona a hombres y mujeres los instrumentos que permiten crear la infraestructura científica esencial para el desarrollo".

El papel de la física en las ciencias de la salud se sintetiza en la biofísica. La biofísica es una rama interdisciplinar de la física con identidad propia que tiene por objeto estudiar los fenómenos biológicos a partir de principios físicos ${ }^{5}$. Así, en la biofísica se estudian, a escala microscópica (nivel molecular) y macroscópica (nivel celular y clínico), los fenómenos físico-biológicos presentes en cualquier tipo de sistema vivo ${ }^{5,6}$.

En una extensa variedad de procesos médicoodontológicos se emplean las leyes de la física para comprender y predecir su curso. Tales leyes también se asocian al desarrollo de tecnologías propias del quehacer clínico ${ }^{5}$. Algunos de los tantos ejemplos de incidencia de la biofísica en salud son: i) explicación y uso de radiación ionizante con fines diagnósticos o terapéuticos en odontología y medicina (concepto de dosis equivalente/efectiva y ley de Bergonié y Tribondeau); ii) leyes de hidrodinámica (Leyes de Bernoulli, de Continuidad y de Poiseuille) para explicar el dolor dental o eventos isquémicos en el cuerpo humano; iii) principios de biomecánica (equilibrio rotacional y traslacional) para establecer límites de elasticidad o rotura en el esqueleto humano, en el diente y materiales restaurativos así como para explicar el movimiento de estructuras corporales y dentoalveolares por medio de los conceptos de fuerza, momento y la relación 
momento de cupla/fuerza; iii) leyes de la termodinámica (ley cero, primera y segunda ley) asociadas con los procesos de transferencias de calor entre el cuerpo y el diente con el ambiente que los rodea; iv) principios de bioelectricidad (potencial eléctrico de Nernst, de membrana y de acción) para explicar el movimiento de iones a través de la membrana de cualquier célula del cuerpo humano, necesario para la transferencia de información. Inclusive, en la actualidad, la biofísica ha contribuido a la lucha contra la pandemia en lo referente al uso de luz ultravioleta $\mathrm{C}$ para la limpieza de superficies y aerosoles contaminados con el coronavirus ${ }^{7}$.

A continuación, se proporcionan dos ejemplos puntuales y sencillos de aplicación de la biofísica en odontología y medicina, respectivamente:

El enfisema subcutáneo es la introducción de burbujas de aire o gas a alta presión en los tejidos suaves del diente vía los espacios del canal radicular o de la membrana dentoalveolar. Dicha burbujas pueden ser generadas por corrientes de aire expelidas por piezas de mano de alta velocidad o jeringas triple. Aunque es una condición clínica poco usual, cuando sucede, puede acarrear complicaciones leves como inflamaciones o, en el peor de los casos, neumomediastino e isquemia ${ }^{8}$.

En la Figura 1 se esquematizan dos modos de secado radicular para preparación del canal radicular. El modo $A$ posee una probabilidad más alta de producción de enfisemas que el $B$ porque la corriente de aire se dirige a la base del canal de la raíz.

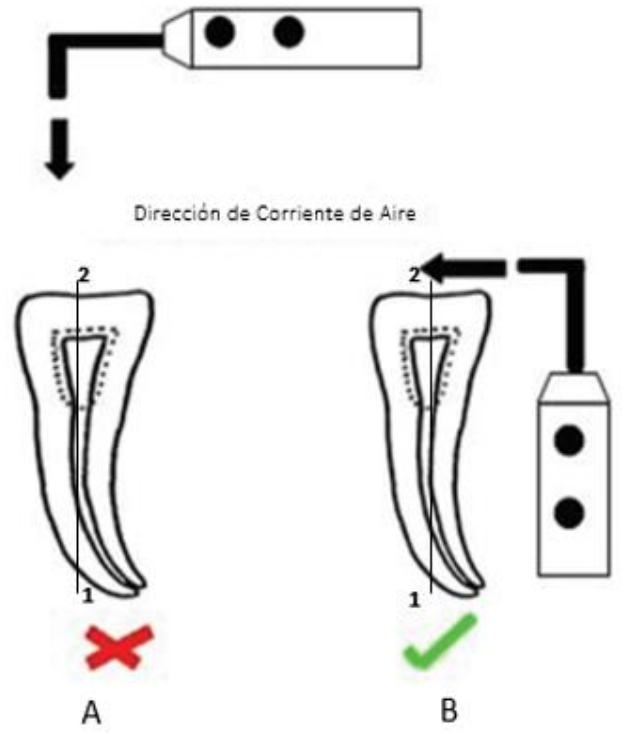

Figura 1. Efecto Venturi en Odontología. Modificada de Shashidhar et $a l^{8}$.

En el modo $B$, no existe riesgo de enfisema porque la corriente de aire no ingresa al canal, sino que se inyecta lateralmente a la cara oclusal del diente. En este caso, se aprovecha el efecto Venturi, un caso particular de la ley de Bernoulli cuando los puntos 1 y 2 se encuentran al mismo nivel de referencia (eje vertical), tal que:

$$
P_{1}+\frac{\rho v_{1}^{2}}{2}=P_{2}+\frac{\rho v_{2}^{2}}{2},
$$

donde $P_{1}$ y $P_{2}$ son las presiones del aire en los puntos 1 y 2 . Para evitar la producción de enfisema, $P_{1}>P_{2}$, de manera que el aire del canal fluya en dirección al plano oclusal. Como en $\mathrm{B}, v_{1}$ es nula dado que no circula aire hacia el canal, la presión en 1 es efectivamente mayor que en 2 , toda vez que,

$$
P_{1}=P_{2}+\frac{\rho v_{2}^{2}}{2}
$$

y su valor aumenta a medida que la velocidad del aire en 2 es incrementada. De este modo, la aplicación de un principio físico evita una condición potencialmente peligrosa en odontología (endodoncia).

La insuficiencia respiratoria aguda es una condición patológica del sistema respiratorio relacionada con la incapacidad para garantizar el intercambio gaseoso a nivel alveolar. Un método no invasivo para lidiar con esta condición es la oxigenoterapia por medio de máscaras de Venturi. La máscara de 
Venturi proporciona una administración controlada de $\mathrm{O}_{2}{ }^{9}$.

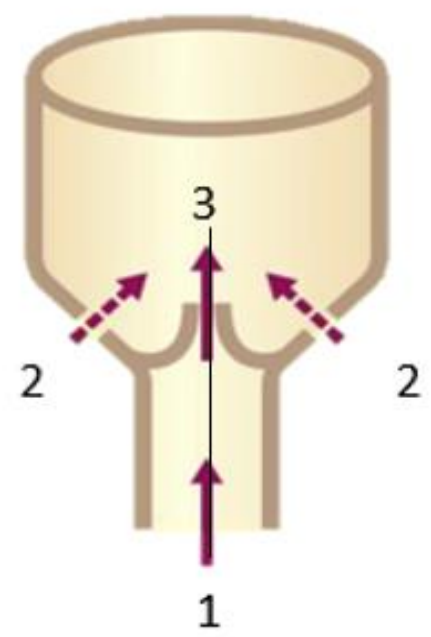

Figura 2. Efecto Venturi en Medicina. Modificada de Mitchell y Cheesmann ${ }^{9}$.

En la Figura 2, se ilustra el principio hidrodinámico de la máscara. En 1 se impulsa $\mathrm{O}_{2}$ puro $(100 \%$ concentrado) por un orificio de alto calibre, el cual al salir del orificio de bajo calibre genera una zona de baja presión en 3 que hace ingresar aire ambiental $\left(\mathrm{O}_{2}\right.$ al $\left.21 \%\right)$ en la máscara mediante orificios en 2 para así disminuir la concentración de $\mathrm{O}_{2}$ a un $26-50 \%$ del total de aire inspirado y que no sea tóxico para el paciente. El efecto Venturi, a lo largo del nivel de referencia (eje vertical), sería:

$$
P_{1}+\frac{\rho v_{1}^{2}}{2}=P_{3}+\frac{\rho v_{3}^{2}}{2}
$$

donde $P_{3}<P_{1}$ siempre que la velocidad del flujo de oxígeno en 3 sea mayor que en 1. Para tanto, por la ecuación de continuidad,

se tiene que,

$$
A_{3} v_{3}=A_{1} v_{1}
$$

$$
v_{3}=\frac{A_{1} v_{1}}{A_{3}}
$$

como el tubo se estrecha en 3 con respecto a 1, o sea $A_{3}<A_{1}, v_{3}$ es consecuentemente mayor que $v_{1}$ creándose así una zona de baja presión en 3 de modo que el aire (a presión atmosférica) atraviesa las ventosas laterales en 2 para mezclarse con el oxígeno puro siguiendo el gradiente de presión (ya que $P_{2}>P_{3}$ ). La concentración de $\mathrm{O}_{2}$ inspirado puede ser ajustada en función del área de los orificios laterales $\left(A_{2}\right)$ por donde ingresa el aire ambiental y por el flujo de $\mathrm{O} 2$ inyectado en 1.

En Colombia, de las 10 mejores universidades en odontología según el Saber Pro 2019, solo 4 poseen, taxativamente, biofísica en su plan de estudios: PUJ, UAM, Unimagdalena y Uninorte. De las 10 mejores universidades en medicina, solo Univalle contempla biofísica (física para ciencias de la salud) otras 3 tienen materias semejantes (biociencias médicas, física básica y biociencias): UIS, Uniandes, Unisabana ${ }^{10}$. De las 10 mejores de enfermería, solo Unitolima tiene biofísica, mientras Univalle, UIS, Unisabana y Unac, materias similares (matemáticas para la salud, biociencias, ciencias básicas y ciencias básicas integradas a los dominios de la enfermería). Demostrado lo apremiante que resulta ser la física en las ciencias de la salud, gran parte de las carreras de medicina, odontología y enfermería deberían tener biofísica en sus planes de estudio como materia desagregada de otras, tal como sucede con bioquímica o biología. No olvidar, además, la incorporación imperativa de un curso de precálculo como prerrequisito, ya que mejoraría la capacidad operativa e interpretativa de los estudiantes de carreras de la salud, así como a reducir el preconcebido estrés relacionado con los "números"5.

Que la "ciencia fundamental" no falte y sea relievada en las facultades de salud del país para que continúen levantándose sobre hombros de gigantes.

\section{REFERENCIAS BIBLIOGRÁFICAS}

1. Giancoli DC. Physics: Principles with Applications. Fifth Edit. Boston, USA: Prentice Hall International; 2016. 1096 p.

2. Feynman RP. The Relation of Physics to Other Sciences. Phys Teach. 1964;2(3):111-7.

3. Holzner. Physics for Dummies. Wiley,John\&Sons, Incorporated. 2005. 64 p.

4. Organización de las Naciones Unidas. Resolución 58293 [Internet]. New York, USA; 2004. 
Disponible

https://undocs.org/en/A/RES/58/293

5. Alonso GL. Biofísica. La ciencia y su enseñanza universitaria. Rev Fac Odontol UBA. 2005;20(49):21-6.

6. Rodas Durán JE. Biofísica: fundamentos e aplicações. First Edit. São Paulo, Brasil: Prentice Hall International; 2003. 318 p.

7. Wilches Visbal JH, Castillo Pedraza MC. Luz ultravioleta lejana para inactivar superficies y aerosoles contaminados con SARS-CoV2. Hacia la Promoción la Salud. 2020;25(2):24-6. Doi: https://doi.org/10.17151/hpsal.2020.25.2.5

8. Shashidhar C, Sarda PN, Parmar J, Patil S. latrogenic periorbital subcutaneous emphysema after endodontic treatment - A case report. Open Access Maced J Med Sci. 2020;8(C):15-8. Doi:
9. Mitchell V, Cheesman K. Gas, tubes and flow. Anaesth Intensive Care Med. 2014;15(5):225-8. Doi: http://dx.doi.org/10.1016/j.mpaic.2017.01.009

10. ICFES. Mejores Universidades de Colombia 2020 [Internet]. Revista Dinero. $2020 \quad$ [citado 13 de octubre de 2020]. p. 1. Disponible en: https://especiales.dinero.com/ranking-delas-mejores-universidades-de-colombia- en2020/index.html.

https://doi.org/10.3889/oamjms.2020.4214 